\title{
Rectoría de la vigilancia en salud pública en el sistema de seguridad social en salud de Colombia: estudio de casos
}

\author{
Yolanda Lucía López¹, Claudia González¹, Berta Natalia Gallego', Ana Lida Moreno² \\ 1 Escuela de Microbiología, Facultad Nacional de Salud Pública, Universidad de Antioquia, Medellín, \\ Colombia \\ 2 Grupo de Química Clínica, Subdirección Red Nacional de Laboratorios, Instituto Nacional de Salud, \\ Bogotá, D.C., Colombia
}

Introducción. Con la reforma del sistema de salud en Colombia, la vigilancia en salud pública continuó siendo una responsabilidad estatal, bajo la rectoría del Ministerio de la Protección Social y las autoridades sanitarias departamentales y locales.

Objetivo. Comprender la manera como los departamentos y municipios han estructurado y desarrollado la vigilancia en salud pública en el marco del sistema general de seguridad en salud en Colombia, posterior a la reforma de 1993.

Materiales y métodos. Se llevó a cabo un estudio cualitativo según el método de estudios de casos múltiples e institucionales en 5 departamentos y 11 municipios, en el año 2004, 10 años después de la reforma en salud.

Resultados. Las autoridades sanitarias han reducido los recursos para asesoría, asistencia técnica, supervisión y control del proceso de la vigilancia en salud pública. Existen deficiencias en aspectos administrativos, la cantidad y calidad del recurso humano, la dotación y los recursos financieros para cumplir las responsabilidades que les han sido asignadas a los departamentos y a los municipios. La vigilancia en salud pública ha priorizado la notificación y el registro de los casos, y su desarrollo ha estado sujeto al interés de algunos funcionarios, principalmente de los hospitales públicos. Existe poco compromiso de los alcaldes, empresas aseguradoras, instituciones prestadoras de servicios de salud y del personal asistencial, para participar en la vigilancia. Falta acción intersectorial para el desarrollo de la vigilancia en salud.

Conclusión. La implementación de la vigilancia en salud pública en el ámbito territorial es débil debido a problemas en la rectoría estatal, manifiestos en la falta de regulación, financiamiento y control en el sistema de salud.

Palabras claves: sistemas de salud, vigilancia de la población, vigilancia epidemiológica, reforma en atención de la salud, seguridad social, regulación y fiscalización en salud, Colombia.

\section{Stewardship of public health surveillance in the health system in Colombia: a cases study}

Introduction. With the reform of the Colombian health system in 1993, public health surveillance continued to be a governmental responsibility under the stewardship of the Ministry of Social Protection along with state and local health authorities.

Objective. The effectiveness of the development and organization of state and municipality public health surveillance programs will be studied as they have evolved under the general social security system in Colombia.

Materials and methods. Qualitative study and case-study method are applied to several institutions as they function in 5 states and 11 counties, ten years after the health system reforms.

Results. Public health authorities have reduced resources for providing advice, technical assistance, supervision and control of the process of the public health surveillance. Weaknesses in administrative control are common. Quantity and quality of human resources, as well as, staffing and financial resources are inadequate to meet the responsibilities that have been assigned to each state and county. The public health surveillance has prioritized the notification and registration of cases, and the strength of development of particular areas occasionally has been subject to the particular interests of officials in charge, particularly in the public hospitals. 
Little commitment or interest is shown by mayors, insurance companies, and institutions providing health services that are supposed to be involved with monitoring. A lack of cross-institutional collaboration is apparent in the development of health services surveillance.

Conclusion. The implementation of public health surveillance at state and local levels is weak due to problems with the governmental stewardship. These weaknesses are manifested in the lack of regulation, financing and control of the health system.

Key words: health systems, population surveillance, epidemiologic surveillance, health care reform, social security, health regulation and fiscalization, Colombia.

En Colombia, las funciones de vigilancia y control epidemiológico fueron reglamentadas desde el antiguo Sistema Nacional de Salud, que funcionó entre 1975 y 1993, y se encontraban bajo la responsabilidad del Ministerio de Salud, las instituciones y los funcionarios del sector público.

Con la Ley 10 de 1990, la Constitución Nacional de 1991 y la Ley 60 de 1993, se descentralizó en el país el sistema de salud y se otorgaron competencias y recursos a los municipios y departamentos; a los municipios se les asignó la responsabilidad en el servicio de salud en el primer nivel de atención y, a los departamentos, en el segundo y tercer nivel, incluyendo las acciones de salud pública. Luego, bajo el contexto de las reformas en salud en Latinoamérica, la Ley 100 de 1993 creó el Sistema General de Seguridad Social en Salud, dejando atrás el Sistema Nacional de Salud, y estableció una nueva normatividad en salud fundamentada en el aseguramiento de la población, donde la rectoría en materia de políticas, normas técnicas y programas y responsabilidades de salud pública, en general, continuó siendo competencia del Estado, bajo la dirección del Ministerio de Salud, en la actualidad Ministerio de la Protección Social. Las funciones administrativas y en salud pública fueron transferidas a las secretarías departamentales, municipales o locales de salud. Así, las funciones de salud pública

\section{Correspondencia}

Yolanda Lucía López, Escuela de Microbiología, Facultad Nacional de Salud Pública, Universidad de Antioquia, Calle 62 № 52-59, Medellín, Colombia.

Teléfonos: (054) 219 6869, (054) 219 6850; fax (054) 219 6850.

yola@guajiros.udea.edu.co

Recibido: 30/01/09; aceptado:12/05/09 fueron incluidas en el plan de atención básico que contiene los servicios de salud dirigidos a la promoción de la salud, prevención de la enfermedad, vigilancia epidemiológica, control de las enfermedades endémicas y epidémicas, control de los factores de riesgo en salud, control de vectores, saneamiento ambiental y eventos con altas externalidades, como las enfermedades transmisibles, entre otras.

En 1999, el actual Ministerio de la Protección Social implementó el software SIVIGILA (Sistema de Vigilancia en Salud Pública), con el fin de registrar los eventos de vigilancia epidemiológica objeto de notificación obligatoria, y cambió el término a vigilancia en salud pública, que se define como un proceso continuo de recolección, análisis e interpretación de los datos para ser usados en planeación, implementación y evaluación de la práctica de salud pública, para una toma adecuada de decisiones políticas, técnicas y administrativas que permitan una intervención eficiente de la problemática en salud $(1,2)$.

La vigilancia en salud pública requiere de un sistema funcional que permita proporcionar información para la toma de decisiones, que involucra el establecimiento de prioridades, decisiones políticas, planificación, ejecución, asignación y movilización de recursos, y la predicción, detección temprana, prevención y control de las epidemias. La vigilancia de enfermedades es, por lo tanto, un componente crítico del sistema de salud para la prestación óptima de la atención en salud y para el desarrollo de estrategias de salud costo-efectivas (3).

Posteriormente, la Ley 715 de 2001 que derogó la Ley 60 de 1993, continuó transfiriendo recursos financieros a los municipios para asumir las 
responsabilidades en salud pública y, dentro de ésta, para la vigilancia en salud pública.

Con la implementación de la Ley 100 de 1993, el Ministerio de Salud (4) promulgó que la vigilancia en salud pública había tenido tropiezos a raíz de los cambios en la organización, estructura y logística del sistema de salud, poca claridad frente al funcionamiento y financiación de la vigilancia, incertidumbre frente a las funciones de los laboratorios de salud pública y desconocimiento de las funciones y actividades de los actores del sistema de seguridad social en salud. Entonces, se desarrolló este estudio motivado por el Instituto Nacional de Salud, como cabeza de la Red Nacional de Laboratorios y coordinador de la vigilancia en salud pública en el país, con el fin de comprender la manera como los departamentos y municipios han estructurado y desarrollado las competencias en esta vigilancia, en el marco del sistema general de seguridad social en salud en Colombia, posterior a la reforma en salud de 1993.

\section{Materiales y métodos}

Se realizó un estudio cualitativo según el método de estudios de casos múltiples e institucional (5-7) sobre la vigilancia en salud pública en Colombia.

El universo fueron los 30 departamentos del país con sus municipios, y la muestra y muestreo fueron por conveniencia, correspondiente a cinco departamentos ubicados en diferentes regiones, y 11 municipios, que fueron los casos.

El criterio para la selección de los departamentos correspondió a aquéllos que tenían el laboratorio departamental de salud pública con mayor desarrollo en la región, puesto que permitía suponer un mayor apoyo para la vigilancia en salud pública de los eventos de interés en salud y, por lo tanto, menos dificultades para el desarrollo de la vigilancia.

En cada departamento, se seleccionaron dos municipios caso, con los siguientes criterios: un municipio con prestación de servicios de salud de primer nivel y, el otro, con segundo nivel, que tuvieran por lo menos una Empresa Social del Estado Hospital y perfil epidemiológico con registro de eventos objeto de vigilancia en salud pública. En algunos de los municipios seleccionados con Empresa Social del Estado Hospital de segundo nivel, se encontró la existencia de otra de primer nivel que, también, se incluyó en el estudio. En el cuadro 1 y la figura 1 se indican los departamentos y municipios estudiados.

Las unidades de análisis fueron: a) oficina que tenía a su cargo la vigilancia en salud pública en la Secretaría Departamental de Salud; b) funcionarios que tenían a cargo la vigilancia en salud pública municipal en la Secretaría Local de Salud o en la Empresa Social del Estado Hospital. En este artículo, estas últimas se nombrarán como hospitales.

Las categorías estuvieron relacionadas con las competencias asignadas por la normatividad para la vigilancia departamental y municipal como: dependencia administrativa que tiene a cargo la vigilancia en salud pública, personal con funciones en dicha vigilancia, nivel de complejidad, proceso técnico-administrativo de la vigilancia en salud pública, recursos, capacitación y manejo de la información, cobertura de notificación, diagnóstico de eventos, funcionamiento del Comité de Vigilancia Epidemiológica, dificultades, entre otras.

Cuadro 1. Departamentos y municipios casos para la vigilancia en salud pública, Colombia, 2004.

\begin{tabular}{lccc}
\hline Región & Departamento & $\begin{array}{c}\text { Municipios caso } \\
\text { Primer nivel }\end{array}$ & $\begin{array}{c}\text { Municipios caso } \\
\text { Segundo nivel }\end{array}$ \\
\hline Atlántica & Córdoba & Lorica, Planeta Rica & Planeta Rica \\
Central & Antioquia & La Estrella, Bello & Bello, Yolombó \\
Oriental & Boyacá & Se Leyva, Sogamoso & Sogamoso \\
Orinoquia y Amazonia & Meta & Puerto López & Acacías \\
Suroccidente & Huila & San Agustín, Pitalito & Pitalito \\
\hline
\end{tabular}




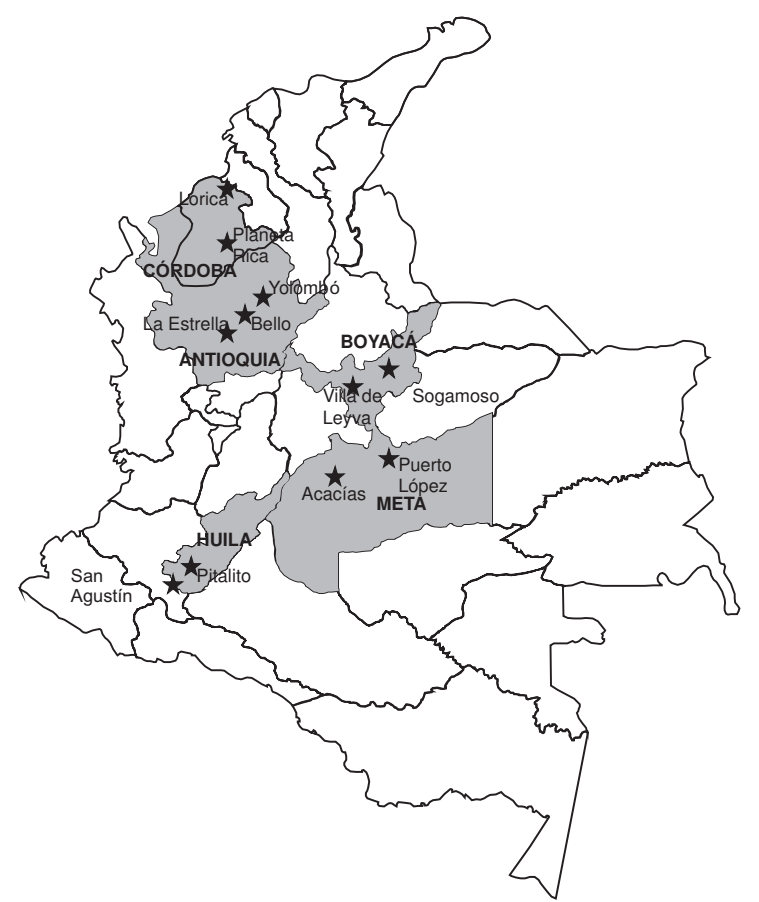

Figura 1. Mapa con los casos de departamentos y municipios

Se elaboró un formato de entrevista con preguntas semiestructuradas, de acuerdo con las categorías anteriores, y se realizó una prueba piloto previa en dos municipios del departamento de Antioquia, con el fin de realizar los ajustes respectivos.

El estudio se realizó mediante entrevistas semiestructuradas y dirigidas a informantes focalizados, como fueron todos aquéllos responsables de las actividades de vigilancia epidemiológica en los entes territoriales y hospitales públicos. Se realizaron 31 entrevistas entre los meses de enero y abril de 2004, así:

- 5 entrevistas a los coordinadores de salud pública o epidemiólogos de las secretarías departamentales de salud,

- 11 entrevistas a los secretarios de salud, coordinadores del Plan de Atención Básica, o responsables de vigilancia en salud pública en las secretarías locales de salud, y

- 15 entrevistas a los coordinadores de vigilancia en salud pública de los hospitales.
Las entrevistas fueron realizadas directamente por los investigadores y grabadas con previa autorización de las secretarías departamentales y locales de salud, hospitales y el consentimiento informado del funcionario.

Se transcribió cada una de las entrevistas y los investigadores procedieron a codificar manualmente las entrevistas mediante el uso de colores, usando un color diferente para cada categoría, el cual correspondió a su código respectivo. Dentro de las categorías se identificaron subcategorías, igualmente codificadas por colores diferentes. Luego, se agruparon las diferentes categorías con sus subcategorías, y se analizó y se escribió cada caso individualmente. Posteriormente, se siguió la metodología de Yin (1994) y Miles y Huberman (1994) (5,6), que consistió en analizar los casos individuales, codificar nuevamente las categorías y subcategorías comunes a todos los casos y aquéllas diferentes, para proceder a describir el cruce de los casos correspondiente a los resultados finales, tal como se presenta en este estudio.

La validación de resultados se hizo por medio de triangulación, así: a) utilización de varias fuentes que corroboraran los hallazgos (funcionarios de secretarías locales de salud y hospitales); b) reunión en cada departamento al finalizar la recolección de información con la participación del secretario de salud y el coordinador de vigilancia en salud pública de la Secretaría Departamental de Salud, los funcionarios entrevistados, el coordinador del Laboratorio Departamental de Salud Pública y el personal investigador, donde se socializaron y validaron los hallazgos generales del estudio en cada localidad; y, c) verificación de los hallazgos con la revisión de fuentes secundarias sobre la teoría acerca de los efectos de la implementación de la Ley 100 de 1993.

Los resultados se consideran aplicables para los casos estudiados.

\section{Resultados}

En el cuadro 2 se presentan las principales convergencias y divergencias encontradas en el estudio de casos. 


\section{Vigilancia en salud pública departamental}

En las secretarías departamentales de salud existe un área que tiene a su cargo la salud pública del departamento, donde la vigilancia en salud pública se rige por las directrices el Ministerio de la Protección Social. No obstante, todas las secretarías departamentales de salud visitadas reconocen que han disminuido las actividades de asistencia técnica, asesoría, supervisión y control a los municipios, debido a la austeridad en el gasto fiscal por el recorte de presupuesto que han sufrido los departamentos. Tal situación conllevó a la reducción del recurso humano, la fusión de los programas de salud pública alrededor del plan de atención básica y disminución de los desplazamientos al terreno e, incluso, en algunas de ellas se eliminaron grupos regionales que tenían a su cargo la supervisión y asesoría en programas de salud pública a los municipios de una región específica del departamento.

"No se visitan los municipios porque los secretarios departamentales [de salud] creen que uno va allá es a pasear, creen que la vigilancia en salud pública se puede supervisar desde el escritorio, y ahora con el comunicado de restricción de gastos es peor", epidemióloga, Secretaría Departamental de Salud.

Cuadro 2. Convergencias y divergencias más notorias con respecto a la vigilancia en salud pública en los casos estudiados.

\section{Convergencias}

Disminución de las actividades de asistencia técnica, asesoría, supervisión y control a los municipios Prioridad en la notificación de los eventos de interés en salud pública

El personal de los departamentos posee perfil ocupacional y experiencia para la vigilancia en salud pública

La alta rotación de funcionarios en los municipios genera dificultades a las Secretarías Departamentales para la vigilancia

Los Laboratorios Departamentales de Salud Pública son valorados por la función que desempeñan en la confirmación de las enfermedades de vigilancia epidemiológica.

El proceso para llevar a cabo la vigilancia en salud pública desde la captación del caso hasta la confirmación y el estudio de campo es muy similar, tanto en los municipios que cuentan con primer nivel como para los de segundo nivel. En ambos, la Secretaría Departamental de Salud es la instancia final para la notificación.

Existen desinterés y escasa respuesta por parte de las Instituciones Prestadoras de Servicios de Salud del sector privado al compromiso de la notificación y la participación en los Comités de Vigilancia Epidemiológica.

\section{Divergencias}

No todas las Secretarías Departamentales tienen equipos para brindar asistencia a los municipios.
Los departamentos más desarrollados tienen mayores medios de comunicación para llevar a cabo la
vigilancia en salud pública.
El personal en los municipios es contratado con frecuencia sin el perfil y experiencia, debido muchas
veces a compromisos políticos.
Sólo los municipios con mayor desarrollo cuentan con un epidemiólogo en la Secretaría Local de Salud.
Sólo aquellas Secretarías Departamentales de Salud que poseen mayor infraestructura y recursos
mantienen actualizados los datos de vigilancia en salud pública por municipio.
La vigilancia en salud pública es asumida en algunos municipios por la Secretaría Local de Salud y en
otros por el hospital público, pero en este último caso, la autoridad sanitaria local no realiza interventoría
a las actividades delegadas al hospital.
Sólo algunos Comités de Vigilancia Epidemiológica Municipales son funcionales y toman decisiones
teniendo como referencia el análisis de los indicadores de vigilancia en salud pública.
En un departamento donde sólo algunos municipios se han descentralizado, la Secretaría
Departamental de Salud aún es la responsable de las acciones de vigilancia en salud pública de aquellos
municipios no certificados en salud.
Tres hospitales de II nivel dejaron las funciones en vigilancia en salud pública municipal y pasaron a
ser sólo unidades notificadoras, lo anterior porque se crearon hospitales de I nivel en los municipios
donde prestaban sus servicios y éstos asumieron la vigilancia en salud pública.


Debido a lo anterior, entre las funciones en vigilancia en salud pública, las Secretarías Departamentales de Salud se han visto obligadas a dar prioridad, casi exclusivamente, a la notificación de los eventos de interés en salud pública y la asesoría a la formulación del Plan de Atención Básico municipal, para lo cual han tratado de fortalecer el trabajo en equipo mediante las Unidades de Análisis o el Comité de Vigilancia Epidemiológica departamental, donde se reúnen epidemiólogos, coordinadores del Plan de Atención Básico y funcionarios del Laboratorio Departamental de Salud Pública, para analizar la información epidemiológica, unificar criterios y tomar decisiones en vigilancia en salud pública.

De acuerdo con las directrices del Ministerio y del Instituto Nacional de Salud, las Secretarías Departamentales de Salud exigen a los municipios la notificación semanal vía radio, teléfono, fax o electrónica; no obstante, una de las dificultades frecuentes es la falta de comunicación durante largos períodos debido a fallas en la red telefónica de los departamentos o municipios; a su vez, la internet es poco disponible.

"Que las oficinas de vigilancia tengan apoyo logístico, un computador, un teléfono, porque generalmente les toca es acomodarse en un ladito que les den", epidemióloga, Secretaría Departamental de Salud.

Para fortalecer la vigilancia en salud pública, las Secretarías Departamentales de Salud brindan talleres de capacitación a los funcionarios de los municipios y cursos de inducción a los profesionales que inician el servicio social obligatorio, pero estas actividades causan desgaste, puesto que existe mucha rotación en el personal debido a que el servicio obligatorio dura seis meses y, con frecuencia, los contratos en las administraciones municipales son inestables debido a que los alcaldes favorecen la contratación por cortos períodos y apenas el funcionario empieza a familiarizarse con su cargo y con el municipio, finaliza el contrato y debe reiniciarse otro proceso de capacitación.

"Supuestamente en este nuevo sistema de salud, se esperaba que las secretarías locales de salud fueran a ser nuestra réplica [de las Secretarías Departamentales de Salud] en los municipios, pero eso no se ha logrado, porque usted no tiene un personal que sea estable en los municipios, porque como son cargos políticos los están moviendo permanentemente", epidemióloga, Secretaría Departamental de Salud.

Por otro lado, a los talleres de vigilancia en salud pública se convoca a quienes tienen esta función en el municipio, es decir, a las secretarías locales de salud pero, en estas instancias, con frecuencia se asigna el cupo a funcionarios que por su perfil profesional o cargo no tienen relación con dicha capacitación y, luego, no la replican en el municipio.

De tal manera, las secretarías departamentales de salud se han visto en la necesidad de identificar dentro de los municipios un interlocutor en el hospital municipal, generalmente el personal de enfermería que tiene a su cargo el manejo de los protocolos de salud pública, desconociendo así la competencia que debe ejercer la secretaría local de salud del municipio en vigilancia en salud pública.

Entre las secretarías departamentales de salud, aquéllas que tienen mayor infraestructura y capacidad de procesamiento de la información mantienen actualizados los datos por municipio pero, en otras, la falta de mayor desarrollo les impide mantener actualizado el perfil epidemiológico departamental y municipal.

"El perfil epidemiológico, no, desgraciadamente no se puede hacer... Lo que se hizo el año pasado fue un análisis completo del SIVIGILA, que eso nunca se había hecho", epidemióloga, Secretaría Departamental de Salud.

El Laboratorio Departamental de Salud Pública ha cumplido una función muy importante en los departamentos para apoyar la vigilancia en salud pública departamental, en lo relacionado con los eventos de interés en salud pública y la vigilancia de los factores de riesgo del consumo y del ambiente.

"El apoyo del Laboratorio Departamental de Salud Pública es oportuno, es una ayuda importante en la salud pública, porque tiene 
pruebas que son diagnósticas para la vigilancia en salud pública", epidemióloga, Secretaría Departamental de Salud.

A su vez, dado que estos laboratorios son unidades notificadoras, una labor importante que realizan algunas secretarías departamentales de salud, y que demanda mucho esfuerzo, es la confrontación de los eventos notificados con los resultados de los exámenes procesados en dichos laboratorios, con el fin de ajustar los datos y contar con información más fidedigna.

\section{Vigilancia en salud pública municipal}

En nueve de los municipios se cuenta con una secretaría local de salud y, en los restantes, con una coordinación en salud o la función es delegada en un secretario del gabinete municipal.

"En los municipios existe un secretario o un director local de salud que muchas veces viene siendo el secretario de planeación o el de gobierno", epidemióloga, Secretaría Departamental de Salud.

No obstante, las secretarías locales de salud no tienen la infraestructura, el recurso humano y la dotación necesaria para asumir las funciones que le competen, pues algunas sólo cuentan con el secretario de salud y otro funcionario. Sólo se encontró un municipio con varios profesionales; sin embargo, la mayoría tiene funciones asistenciales.

El proceso para llevar a cabo la vigilancia en salud pública en los 11 casos, desde la captación del caso hasta la confirmación y el estudio de campo, es muy similar tanto en los municipios que cuentan con primer nivel como para los de segundo nivel. En ambos procesos, la Secretaría Departamental de Salud es la instancia final para la notificación (figura 2). Para el diagnóstico de los eventos, el laboratorio clínico y el Laboratorio

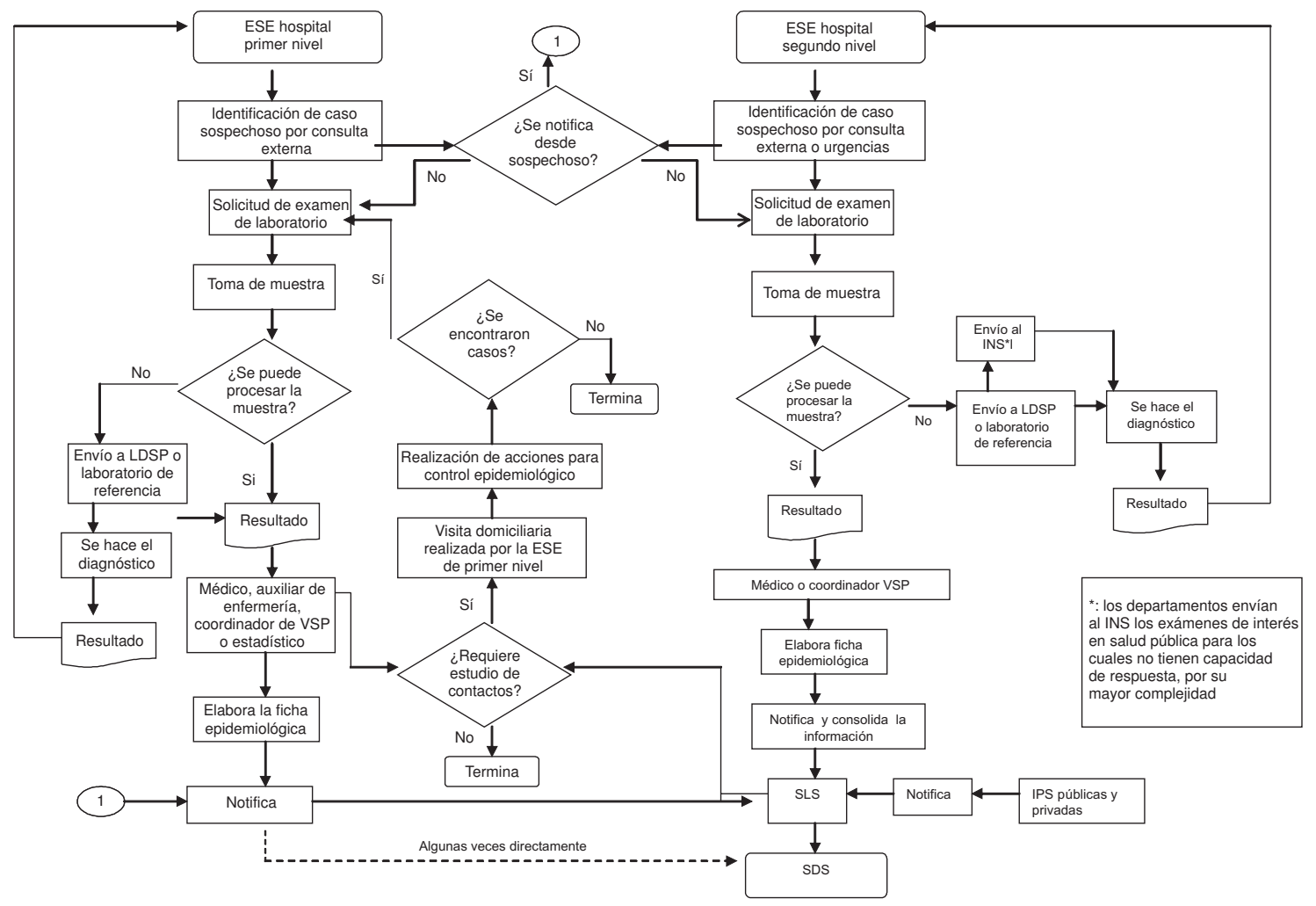

Figura 2. Flujograma de la vigilancia en salud pública de los casos estudiados. 
Departamental de Salud Pública como nivel de referencia, son muy importantes en este proceso.

"Aquí, de todas maneras, hay que reconocer que el laboratorio es una prioridad y es una fortaleza... algunas veces, los médicos olvidan hacer la notificación, pero el laboratorio, al estar también entrenado, entonces también reporta. De esta manera, no se nos pasa por alto un reporte importante", epidemiólogo, Secretaría Departamental de Salud.

Aunque el municipio tenga secretaría local de salud o asuma funciones en salud, no necesariamente implica que éste desarrolle las funciones de vigilancia en salud pública, puesto que algunos las contratan en parte o en su totalidad, con el hospital local que cuenta con recurso humano y experiencia en esta vigilancia, principalmente el personal de enfermería (cuadro 3).

"Nosotros hacemos cada año un contrato con los dos hospitales para la atención de las visitas epidemiológicas de campo..., pues ellos son las personas que desde años atrás tienen la experiencia. La labor mía aquí en la Secretaría Local de Salud es prácticamente administrativa, salvo algunos eventos especiales que se requiera que también yo salga a hacer la visita de campo", epidemiólogo, Secretaría Local de Salud.

No obstante, con frecuencia el municipio no hace interventoría al hospital y, muchas veces, los recursos que le transfiere no son suficientes o, incluso, no existe contrato y el hospital asume voluntariamente las funciones ante el hecho de no estarse realizando la vigilancia en salud pública en el municipio.

"No se le ha exigido al municipio que, así sea por contrato, él esté pendiente de lo que el prestador hace. Entonces, le contrata pero se desentiende totalmente de lo que es responsabilidad suya", epidemióloga, Secretaría Departamental de Salud.

En un departamento donde sólo un pequeño número de municipios se han descentralizado en salud, se encontró que la Secretaría Departamental de Salud aún es la responsable de las acciones de vigilancia en salud pública municipal de aquéllos municipios no certificados en salud $y$, para desempeñarlas, realiza convenios interadministrativos con los hospitales locales o regionales.

Con respecto al nivel de complejidad, en tres de los municipios con hospital de II nivel, que actuaban como sede regional prestando

Cuadro 3. Instancias municipales que asumen la orientación en salud y la vigilancia en salud pública, estudio de casos, Colombia, 2004.

\begin{tabular}{|c|c|c|c|c|c|}
\hline \multirow[b]{2}{*}{ Departamento } & \multirow[b]{2}{*}{ Municipio } & \multirow[b]{2}{*}{$\begin{array}{l}\text { Orientación de } \\
\text { salud municipal }\end{array}$} & \multicolumn{3}{|c|}{ Instancia que asume la vigilancia en salud pública } \\
\hline & & & $\begin{array}{l}\text { Secretaría de } \\
\text { Salud }\end{array}$ & $\begin{array}{l}\text { ESE Hospital } \\
\text { nivel I }\end{array}$ & $\begin{array}{l}\text { ESE Hospital } \\
\text { nivel II }\end{array}$ \\
\hline \multirow[t]{2}{*}{ Huila } & San Agustín & Secretaría de Salud & $\mathrm{X}$ & & \\
\hline & Pitalito & Secretaría de Salud & $\mathrm{X}$ & & \\
\hline \multirow[t]{2}{*}{ Boyacá } & Villa de Leyva & $\begin{array}{l}\text { Secretaría de Desarrollo } \\
\text { Económico y Social }\end{array}$ & & $\mathrm{X}^{*}$ & \\
\hline & Sogamoso & Secretaría de Salud & $x$ & & \\
\hline \multirow[t]{2}{*}{ Meta } & Acacías & Coordinación de salud & & & $\mathrm{X}$ \\
\hline & Puerto López & Secretaría de Salud & & $\mathrm{X}$ & \\
\hline \multirow[t]{3}{*}{ Antioquia } & La Estrella & Secretaría de Salud & $x$ & & \\
\hline & Bello & Secretaría de Salud & $\mathrm{X}$ & $X^{* *}$ & \\
\hline & Yolombó & Secretaría de Salud & $\mathrm{X}$ & & \\
\hline \multirow[t]{2}{*}{ Córdoba } & Planeta Rica & Secretaría de Salud & & $x$ & \\
\hline & Lorica & Secretaría de Salud & $x$ & $x$ & \\
\hline
\end{tabular}

\footnotetext{
${ }^{*}$ Por convenio interadministrativo con el instituto seccional

**La ESE de primer nivel asume los estudios de campo.
} 
servicios asistenciales y de salud pública de primer y segundo nivel a la población de influencia, luego de la descentralización en salud, en estos municipios se creó un nuevo hospital de primer nivel, con el cual la secretaría local de salud contrata la vigilancia de la salud pública y el Plan de Atención Básica. De esta manera, estos hospitales de II nivel dejaron las otras funciones en vigilancia y pasaron a ser sólo unidades notificadoras. Los otros dos hospitales de II nivel estudiados tienen a su cargo, también, el primer nivel en el municipio; uno de ellos tiene a su cargo la vigilancia en la salud pública municipal.

Una de las dificultades que perciben las secretarías locales de salud para la vigilancia en salud pública en el ámbito municipal es el bajo nivel de escolaridad de los alcaldes y concejales, quienes finalmente toman las decisiones para la asignación de recursos y la contratación del personal. De tal manera, aunque algunas secretarías departamentales de salud realizan actividades de capacitación a los aspirantes a alcaldías, cuando inician el nuevo mandato, no es suficiente para que los alcaldes tomen conciencia de sus competencias y bajo el contexto de la autonomía local, nombran a personas que no tienen el perfil idóneo para dirigir la secretarías locales salud y la vigilancia en salud pública.

"Aquí hay mucha gente que para estar de coordinador no tiene ninguna formación en salud, entonces, eso es un inconveniente bastante grande", epidemióloga, Secretaría Departamental de Salud.

"Porque el perfil de los trabajadores de las direcciones locales es muy bajito en el área de la salud, entonces cuando vos les hablás de muchas cosas, uno cree que le están recibiendo la información pero ellos dan la vuelta y al otro día ya no se acuerdan de nada", epidemióloga, Secretaría Departamental de Salud.

Además, las secretarías locales de salud aún no asumen la planeación y las intervenciones en salud con base en el análisis de su propia situación epidemiológica y, la mayoría de ellos, esperan que ante los brotes o emergencias en salud pública, el ente departamental asuma la responsabilidad.

"Los alcaldes requieren una inducción en lo que es salud, porque ellos todavía viven convencidos de que el hospital es el responsable de la salud del municipio", epidemióloga, Secretaría Departamental de Salud.

Tampoco es raro encontrar que las intervenciones en el municipio no obedezcan al perfil epidemiológico, sino que estén encaminadas a satisfacer a los habitantes o a aumentar el nivel de popularidad del gobernante.

"En una visita que hicimos al único foco de malaria autóctono del departamento, vimos que dentro de su Plan Local de Salud para nada habían (sic) actividades con respecto a esta patología. Ellos tenían programadas actividades contra el dengue, fumigación masiva, y no tenían ni un solo caso de dengue", epidemióloga, Secretaría Departamental de Salud.

Con respecto al Comité de Vigilancia Epidemiológica, en los municipios se encontraron diferentes niveles de cumplimiento, como se muestra en el cuadro 4. Lo más común es que el comité del hospital reemplace al del municipio porque es más funcional.

Las Secretarías Departamentales de Salud perciben que la mayoría de los Comités de Vigilancia Epidemiológica municipales no se reúnen con la periodicidad que tienen establecida, falta claridad sobre las funciones de los miembros que los conforman y existe inexperiencia e inestabilidad en los cargos; de ahí que consideran que necesitan ser reformulados, puesto que normalmente no toman decisiones basadas en el análisis de la información epidemiológica y con una visión de planificación de la salud municipal.

Por otro lado, aunque, en general, la cobertura de notificación de los eventos de interés en salud pública en los municipios y departamentos cumplen con la cobertura mínima establecida por la autoridad sanitaria nacional, sólo algunas instituciones trascienden el conteo de los casos y realizan consolidados y análisis periódicos de la situación de salud municipal, por lo que es común 
Cuadro 4. Comités de vigilancia epidemiológica (COVE) por municipio, estudios de caso, Colombia, 2004.

\begin{tabular}{llll}
\hline $\begin{array}{l}\text { Departamento } \\
\text { y municipio }\end{array}$ & COVE municipal & Características de los covE & COVE institucional
\end{tabular}

$\begin{array}{lll}\text { Huila } & \begin{array}{l}\text { Poca claridad en sus funciones, alta rotación } \\ \text { San Agustín quienes lo conforman, ausencia de análisis } \\ \text { de la situación epidemiológica del municipio. } \\ \text { Dos reuniones en el 2003. }\end{array} & \begin{array}{l}\text { Se analiza la información de la vigilancia en salud pública } \\ \text { para desarrollar el PAB que tienen contratado por la SLS. } \\ \text { Reuniones cada dos meses. }\end{array} \\ & \end{array}$

Pitalito Se enfoca en resolver problemas de diligenciamiento de las fichas de notificación. No existe la ESE de segundo nivel de complejidad. La SLS reporta nueve reuniones en el 2003, pero la ESE local fue convocada solo a tres reuniones.

\section{Boyacá}

Villa de Leyva No se reúne hace dos años.

Sogamoso Tiene representatividad de todas las IPS del municipio, se hace autocapacitación en vigilancia en salud pública, se revisan las políticas en salud, se estudian casos, se presentan los informes de vigilancia en salud pública y se programan actividades. Tiene periodicidad mensual.

Meta

Acacías y No existe COVE municipal como tal, sino que Puerto López la SLS participa en las reuniones del COVE institucional de la ESE.

\section{Antioquia}

La Estrella

Bello

Yolombó
Los tres municipios tienen COVE con representatividad de todas las IPS del municipio, de otros sectores y, algunas veces, de la comunidad. En las reuniones se hace autocapacitación en vigilancia en salud pública, se revisan las políticas en salud, se estudian los casos, se presentan y analizan los informes de vigilancia en salud pública, se toman decisiones y se programan actividades. La periodicidad es cada dos meses, pero en Bello no se reunió en el 2003

\section{Córdoba}

Planeta Rica No existe COVE municipal como tal, sino que la SLS participa en las reuniones del COVE institucional de la ESE.

Lorica No existe COVE municipal, sin embargo, las epidemiólogas de las dos ESE y una profesional universitaria de la SLS mantiene comunicación permanente para definir las actividades de vigilancia en salud pública y control de patologías.
La ESE de primer nivel no tiene COVE, pro existe articulación entre el área asistencial y el equipo que realiza la vigilancia en salud pública. La ESE de segundo nivel reactivó el COVE en el 2003, después de cinco años de estar cesante porque se priorizaron las funciones asistenciales del personal.

Cada ESE (primer nivel en los municipios y segundo nivel en Sogamoso) tienen COVE, en el cual se realiza análisis de la vigilancia en salud pública y de saneamiento ambiental municipal, se hace autocapacitación en vigilancia en salud pública y se programan acciones colectivas para control epidemiológico. La periodicidad de reuniones es cada uno o dos meses para las ESE de primer nivel y sin una regularidad precisa en la ESE de segundo nivel.

Énfasis en análisis de la notificación y casos epidemiológicos puntuales. Ausencia de análisis integral de vigilancia en salud pública municipal y falta de periodicidad en reuniones, puntuales. Ausencia de análisis integral de vigilancia en salud planeación y evaluación de actividades.

Todas las ESE (de primer nivel para los tres municipios y de segundo nivel para Yolombó y Bello) tienen conformado el COVE institucional, que se reúne cada dos meses. Tiene una dinámica similar en el análisis, autocapacitación, toma de decisiones y planificación similar a la descrita en los COVE municipales, incluyendo también, el análisis de las infecciones intrahospitalarias. La periodicidad es de cada dos meses.

La periodicidad de las reuniones obedece al análisis de casos epidemiológicos puntuales, hay ausencia de análisis integral de vigilancia en salud pública municipal, falta periodicidad en reuniones, planeación y evaluación de actividades.

Su periodicidad es cada dos meses y en él analizan la situación de morbilidad atendida a nivel institucional, se elaboran planes de trabajo y se asignan tareas. 
encontrar que en la secretaría local de salud no se cuenta con un documento actualizado sobre el perfil epidemiológico del municipio.

En un municipio se encontraron dificultades en la puesta en operación de la vigilancia en salud pública, debido a las disputas del pasado entre los directivos de la secretaría local de salud y los del hospital y, en otros municipios, la interacción entre estas dos instancias no se encontró debido a riñas políticas entre éstos.

\section{Vigilancia en salud pública y el personal de salud}

Con frecuencia, se tiene la percepción de que la vigilancia en salud pública en el ámbito institucional depende de la importancia que le otorgue el gerente y el coordinador científico del hospital, puesto que no se considera una labor inherente a la prestación de servicios de salud. Además, existe desconocimiento y poco interés en los funcionarios sobre los protocolos de vigilancia en salud pública, lo cual hace que se presenten fallas en el manejo de los eventos objeto de vigilancia en salud pública, con todas las consecuencias que puede traer para la población, el no detectarlos y controlarlos oportunamente.

Los funcionarios presentan carencias desde la formación de pregrado, razón por la cual pocas personas se comprometen con esta actividad; muchas veces los registros son llenados como un requisito y el personal médico no tiene interés en actualizarse en los protocolos y, mucho menos, en diligenciar de manera adecuada las fichas para reportar los eventos de notificación obligatoria. Además, la carencia de mecanismos efectivos de vigilancia y control conlleva a desgaste en los coordinadores de vigilancia en salud pública y de los Comité de Vigilancia Epidemiológica, tratando de complementar la información que deberían tener los registros y las fichas epidemiológicas.

\section{Acción interinstitucional e intersectorial}

Otra dificultad que expresan los coordinadores de vigilancia en salud pública, es la falta de participación del sector privado y la actitud poco interesada y escasa respuesta por parte de las
Instituciones Prestadoras de Servicios de Salud al compromiso de la notificación y la participación en los Comités de Vigilancia Epidemiológica municipales, dado que éstos siempre tienen la menor cobertura en la notificación.

“... no podemos trabajar nosotros solos aquí en el hospital, si el municipio no se interesa por coordinar con las otras instituciones", coordinadora, Vigilancia en Salud Pública, hospital de primer nivel.

\section{Discusión}

Se destaca que las autoridades sanitarias departamentales en el país manifestaron haber reducido los recursos para asesoría, asistencia técnica y supervisión del proceso de la vigilancia en salud pública en los entes locales, funciones que son competencia del nivel estatal.

De manera similar, en los municipios se encontraron deficiencias en lo referente a aspectos administrativos, cantidad y calidad del recurso humano, dotación y recursos financieros en las administraciones municipales para asumir las responsabilidades que les compete para garantizar la vigilancia en salud pública en el ente territorial.

Esta situación, además de la prioridad que ha sido dada por las secretarías departamentales de salud para recibir la notificación de los eventos de interés en salud pública en las sedes centrales, debido a las dificultades para ejercer funciones complementarias en el terreno, permite evidenciar una disminución de las responsabilidades y competencias en la conducción, regulación y vigilancia de los servicios de salud que son propias e indelegables del Estado. Circunstancias análogas fueron descritas para el control de la tuberculosis en el país, donde se reportó disminución en los recursos humanos y en la planeación, inspección, vigilancia y control, tanto en el ámbito departamental como en el local $(8,9)$.

Lo anterior ocurrió después de la reforma del sistema de salud en el país con la Ley 100 de 1993, que se implementó paralelamente a las reformas políticas, administrativas y fiscales de ajuste estructural en la década del 90 y produjeron 
la reducción del tamaño y rol del Estado, con limitaciones financieras que obligaron a reducir los recursos humanos y a implementar medidas de austeridad en los gastos de funcionamiento de municipios y departamentos.

Esta escasez de recursos en el ámbito municipal para el desarrollo de la vigilancia en salud pública es contradictoria con el incremento de los recursos que han sido asignados para el aseguramiento en salud, después de la reforma (10). De acuerdo con la investigación realizada por Gilberto Barón, en el período 1998-2002, el gasto total en salud en relación con el producto interno bruto (PIB) fue en promedio de $8,5 \%$, en comparación con el año 1993, cuando fue de $6,2 \%(11)$.

De tal manera, los municipios no cuentan con los recursos humanos, físicos y financieros suficientes para asumir las responsabilidades conferidas por la ley, problema que ha sido ampliamente detectado en los procesos de reforma y descentralización implementados en América Latina (11), en este caso, en salud pública y en vigilancia en salud pública dadas por la Ley 715 de 2001. Como consecuencia de ello, varios autores han registrado un efecto negativo sobre la salud pública con deterioro progresivo en el cumplimiento de las funciones $y$ un retroceso en la vigilancia y el control epidemiológico en el sistema de salud, con subregistro de las principales enfermedades de interés en salud pública, falta de aplicación de los protocolos y guías de atención y resurgimiento de enfermedades y brotes epidémicos, como rabia animal, tuberculosis, fiebre amarilla, entre otras, como resultado de un modelo de reforma fundamentado en la atención sanitaria individual y las fuerzas del mercado para la prestación de los servicios de salud (12-16).

Se encontró, además, que la vigilancia en salud pública se ha mantenido prioritariamente en el modelo de la vigilancia epidemiológica, entendida ésta como el proceso regular y continuo de observación e investigación de las principales características y componentes de la morbilidad, mortalidad y otros eventos en salud (Decreto 1562 de 1984), que se desarrolló en el contexto del antiguo Sistema Nacional de Salud y que con la reforma en salud a partir de la Ley 100 de 1993, ha continuado desarrollándose principalmente por los funcionarios de los hospitales, debido a la falta de capacidad en las secretarías locales de salud de los municipios para ejercerla.

En esta dirección, la vigilancia en salud pública se ha centrado en la notificación de eventos, principalmente de morbilidad, tal como se tenía en el contexto de la vigilancia epidemiológica, situación que se evidencia no sólo en las entrevistas sino también en los flujogramas del proceso de tal vigilancia; asimismo, una fortaleza para el apoyo a la vigilancia en salud pública la constituyen los Laboratorios Departamentales de Salud Pública que, a pesar de ser entidades públicas y estar sujetos a las mismas restricciones en la disminución de recursos humanos, físicos y financieros para su funcionamiento (17), los municipios consideran que han continuado recibiendo el apoyo con exámenes de laboratorio para el diagnóstico de eventos de interés en salud pública establecidos en la normatividad y, a su vez, las Secretarías Departamentales de Salud utilizan esta información para confirmar los casos, retroalimentar el Sistema de Vigilancia Epidemiológica, SIVIGILA, y construir el perfil epidemiológico.

A su vez, puede deducirse que, entre las acciones de salud pública dentro del plan de atención básica, los municipios toman la decisión de no invertir o invertir poco en la vigilancia en salud pública y priorizan las acciones dirigidas a la prevención de la enfermedad, generalmente dispersas y no articuladas a la prestación de los servicios, dejando con frecuencia la responsabilidad en los hospitales, debido a que las Secretarías Departamentales de Salud no están ejerciendo la función de regulación en los municipios para fiscalizar el cumplimiento de la normatividad en relación con la vigilancia en salud pública. En este sentido, esta vigilancia no ha trascendido a otros desarrollos en salud pública, a pesar de que el Ministerio de la Protección Social estipuló que dicha vigilancia debía trascender al proceso de análisis, interpretación y divulgación de la información 
para orientar y generar acciones de promoción de la salud, prevención y control, como una de las funciones esenciales de la salud pública para el mejoramiento de las condiciones de salud de la población $(2,18,19)$.

Los resultados indican, además, dificultades en el manejo de la información epidemiológica en los municipios y departamentos, puesto que no se tienen directrices estandarizadas para la consolidación, análisis y representación de la información en salud pública, dejándose a la propia decisión y capacidad técnicoadministrativa de cada ente territorial. En tal sentido, debe destacarse que, aunque el software SIVIGILA ha constituido un aporte esencial para registrar y notificar en red los eventos objeto de vigilancia en salud pública en el territorio nacional, aún se tienen dificultades de acceso a este medio de comunicación y la información ha sido fragmentada, porque no se encuentra articulada al diagnóstico de la situación de salud y perfil epidemiológico de las localidades; con frecuencia, los alcaldes desconocen la situación de salud en su territorio.

Esto indica que existen fallas en la conducción del sistema de información en salud y que la reforma no superó la fragmentación de este sistema. Esta situación ha sido documentada como uno de los principales talones de Aquiles y grave consecuencia de la reforma en salud en el país, puesto que no existe información actualizada y confiable sobre la situación epidemiológica de la población, la confiabilidad varía según la región y se desarticuló el sistema de información, a excepción de la información provista por el SIVIGILA para las enfermedades de notificación obligatoria $(10,20)$.

A pesar de las debilidades, quizá la notificación en el SIVIGILA se ha constituido en uno de los principales testigos que permiten documentar los efectos de la reforma y del corte en el financiamiento del sistema de salud en los programas e indicadores de salud pública, como un papel activo que han cumplido los epidemiólogos y otros trabajadores de la salud pública para hacer frente a las reformas anti-salud, y que se evidencia en el registro emergente de enfermedades transmisibles e inmunoprevenibles y las bajas coberturas de vacunación en el país. Asimismo, es importante tener en cuenta que el enfoque epidemiológico es una herramienta que permite planear la salud con acciones costo-efectivas para reducir no sólo los factores de riesgo, sino también la morbimortalidad (21-23).

Por otro lado, la regulación dentro de la función rectora en el sistema de salud no ha definido los perfiles de formación que debe exigirse a los funcionarios que desempeñan los cargos de dirección en las secretarías locales de salud y, menos aún, de los funcionarios que desempeñan las actividades de vigilancia en salud pública. Tal situación ha conllevado a que estos cargos sean desempeñados por algún técnico o profesional del área de la salud o de otra área, sin formación en el campo de la salud pública o la epidemiología, y sin un control por parte de las autoridades sanitarias departamentales o del Ministerio de la Protección Social.

De tal manera, la reforma en el sistema de salud no ha producido una acción sinérgica de expansión a la protección de la salud fundamentada en las funciones esenciales de salud pública y con los perfiles de formación y desempeño del recurso humano adecuado a la práctica de la salud pública, puesto que el sistema ha dado prioridad al aseguramiento en salud. Los estudios en recurso humano han documentado que más de $90 \%$ se desempeñan en la prestación de los servicios de salud del sistema general de seguridad social y entre $5,3 \%$ y $12 \%$, en servicios de salud pública, vigilancia y control $(24,25)$. Esta situación se refleja en la formación del recurso humano en las universidades donde se da prioridad de la formación clínica sobre la formación bajo un enfoque de atención integral en salud al paciente basado en la salud pública, prevención de la enfermedad y promoción de la salud, lo cual se ha revertido en la práctica profesional en las instituciones de salud con el poco compromiso que adquieren para vincular las funciones de la vigilancia en salud pública en la prestación de los servicios de salud.

Puede decirse, entonces, que la vigilancia en salud pública, de manera similar a la forma 
como ha sido implementada la reforma en salud en el país, no responde a los principios de la Ley 100 de 1993 del Sistema de Salud, lo que se evidencia en que: no existe universalidad con cobertura total de las acciones debido a la disminución de las funciones por parte de los municipios y departamentos; no hay equidad ni integridad debido a que privilegia la atención asistencial clínica a la atención integral en salud, discriminándose la vigilancia en salud pública; no posee calidad porque aún existe un gran desconocimiento de la función esencial de la vigilancia en salud pública en el sistema y en el recurso humano en salud. En tal sentido, los programas de salud pública que se consideraban una fortaleza en el Sistema Nacional de Salud anterior, se debilitaron por el proceso de descentralización y la reforma de la salud, a pesar de que éstas pretendían desarrollar más las capacidades para la formulación de políticas, seguimiento, evaluación y asistencia técnica en salud (26). La Organización Panamericana de la Salud documentó que en América no se tiene un total conocimiento de las funciones de la vigilancia en salud pública (27).

Igualmente, las empresas aseguradoras en salud y las instituciones prestadoras de servicios de salud privadas han tenido poco compromiso y participación en la vigilancia en salud pública y en los Comités de Vigilancia Epidemiológica departamentales y municipales, dejando en el sector público la responsabilidad del cumplimiento de las funciones de dicha vigilancia, sin ningún control por parte de las autoridades sanitarias.

Por otro lado, las problemáticas de salud en los entes departamentales y municipales aún no se plantean desde un punto de vista intersectorial, dejando en cabeza de las secretarías departamentales y locales de salud y hospitales, la respuesta no sólo a las actividades de la vigilancia en salud pública sino a la intervención de los problemas detectados, con enfoque sectorial. En tal sentido, los sectores de educación, desarrollo comunitario, ambiental, agropecuario y judicial, entre otros, continúan ausentes en los Comités de Vigilancia Epidemiológica y, por ende, en la participación del análisis e intervención de las problemáticas comunes para mejorar la salud de las poblaciones.

El presente estudio tuvo la limitación de describir los procesos de la vigilancia en salud pública en sitios cercanos a las capitales de los departamentos seleccionados, lo cual podría evidenciar una mejor respuesta a la vigilancia en salud pública que en municipios más alejados y con mayores problemas de acceso a los servicios de salud. A su vez, sólo se estudiaron las autoridades sanitarias y los hospitales públicos, faltando describir la situación en las instituciones prestadoras de servicios de salud privadas.

Se concluye que la vigilancia en salud pública en el ámbito municipal es débil debido a la falta de rectoría en el sistema de salud, mediante el ejercicio de las funciones de regulación, conducción, financiamiento y prestación del servicio de salud, en el sistema general de seguridad social en salud en Colombia.

\section{Agradecimientos}

A los jefes y coordinadores de vigilancia en salud pública, epidemiólogos y jefes de los Laboratorios de Salud Pública de las Secretarías Departamentales de Salud de Antioquia, Huila, Meta, Córdoba y Boyacá, por los valiosos aportes e información que nos brindaron para este estudio.

A los secretarios de salud, coordinadores de vigilancia en salud pública y funcionarios de las Secretarías Locales de Salud y ESE Hospitales de los municipios de Bello, Yolombó, La Estrella, Pitalito, San Agustín, Puerto López, Acacías, Lorica, Planeta Rica, Villa de Leyva y Sogamoso, por los valiosos aportes e información que nos brindaron para este estudio.

\section{Conflicto de intereses}

Los autores del artículo hacen constar que no existe, de manera directa o indirecta, ningún tipo de conflicto de intereses que puedan poner en peligro la validez de lo comunicado.

\section{Financiación}

Este trabajo fue financiado por la Red Nacional de Laboratorio del Instituto Nacional de Salud. 


\section{Referencias}

1. Thacker SB, Berkelman RL. Public health surveillance in the United States. Epidemiol Rev. 1988;10:164-90.

2. Ministerio de Salud. La vigilancia en salud pública de eventos transmisibles y de fuente común. Tomo 1. Documentos generales y protocolos de enfermedades transmitidas por alimentos. Santafé de Bogotá: Ministerio de Salud; 2000. p 1-29.

3. Organización Panamericana de la Salud. Un enfoque integrado para la vigilancia de las enfermedades transmisibles. Boletín Epidemiológico. 2000;21:1-4.

4. Ministerio de Salud. El papel de los laboratorios de salud pública en la vigilancia de la salud pública. Inf Quinc Epidemiol Nac. 1999;4:259-67.

5. Yin R. Case study research: Design and methods. London: Sage publications; 1994. p. 171.

6. Miles M, Huberman M. Qualitative data analysis California: Sage publications; 1994. p. 338.

7. Stake R. Case studies. En: Denzin N, Lincoln Y, editors. Handbook of qualitative research. California: Sage Publications; 1994. p. 236-47.

8. Arbeláez MP, Gaviria MB, Franco A, Restrepo R, Hincapié D, Blas E. Tuberculosis control and managed competition in Colombia. Int $\mathrm{J}$ Health Plann Manage. 2004;19:25-43.

9. Arbeláez MP. La reforma del sector salud y el control de la tuberculosis en Colombia. En: Descentralización y gestión del control de las enfermedades transmisibles en América Latina. Buenos Aires: OPS; 2006. p. 11529.

10. CEPAL. Ensayos sobre el financiamiento de la seguridad social en salud. Los casos de Estados Unidos, Canadá, Argentina, Chile, Colombia. Volumen II. Santiago de Chile: Fondo de Cultura Económica; 2000. p. 813.

11. Barón G. Cuentas de salud de Colombia 1993-2003: el gasto nacional en salud y su financiamiento. Bogotá: Ladiprint Editorial Ltda.; p. 155.

12. Saenz O. Impacto de la crisis económica y las políticas neoliberales sobre la salud colectiva en América Latina. En: Cardona A, editor. Sociedad y Salud. Bogotá: Ed. Zeuss Asesores Ltda; 1992. p. 107-26.

13. Franco S. Hacia una política nacional de salud pública y balance de la reforma colombiana. Cartilla de Seguridad Social. 1998;5:71-90.

14. Franco A. Referente para el análisis de la situación de salud pública. Revista Nuevos Tiempos. 2002;10:101-8.
15. Academia Nacional de Medicina. Ley 100 de 1993: reforma y crisis de la salud. Parte II. Bogota: Ed. Kimpres Ltda; 2002. p. 217.

16. Jaramillo I. El futuro de la salud en Colombia. Ley 100 de 1993. Cinco años después. Santafé de Bogotá: FESCOL, FES, Fundación Corona, Fundación Antonio Restrepo Barco; 1999. p. 405.

17. López YL, González MC, Escobar JP, Chávez, BM. Los laboratorios de salud pública en el sistema general de seguridad social en salud, Colombia, 2000. Estudio de casos. Revista Facultad Nacional de Salud Pública. 2003;21:9-25

18. Muñoz $F$, López-Acuña $D$, Halverson $P$, Guerra de Macedo C, Hanna W, Larrieu M, et al. Las funciones esenciales de la salud pública: un tema emergente en las reformas del sector de la salud. Rev Panam Salud Pública. 2000;8:126-34

19. Mantilla G, González M, Espinosa J. Lineamientos para el sistema de vigilancia y control en salud pública. Inf Quinc Epidemiol Nac. 2005;10:369-78.

20. Cardona A, Estrada A, Nieto E, Agudelo B, Chávez BM, Montoya A, et al. Impacto de la reforma de la seguridad social sobre la organización institucional y la prestación de los servicios de salud en Colombia. Medellín: Universidad de Antioquia, Facultad Nacional de Salud Pública; 1999. p. 297.

21. Lalonde M. A new perspectiva on the health of Canadians. Ottawa: Government of Canada; 1974.

22. Terris M. Epidemiología y política de salud en las Américas: el desafío neoliberal. Revista Facultad Nacional de Salud Pública. 2004;14:79-89.

23. Ruales J. Desafíos y perspectivas de los sistemas de salud y la gestión frente a las funciones esenciales de la salud pública. Revista Facultad Nacional de Salud Pública. 2004;22(número especial):21-32.

24. Ministerio de Salud, Universidad de Antioquia. Recursos humanos en salud en Colombia, 1961-2005. Medellín: Tecnopress; 2003. p. 190.

25. López JG. Oferta y demanda de recursos humanos en salud. Revista Facultad Nacional de Salud Pública. 2004;22:93-102.

26. Yepes FJ, Sánchez LE. Las últimas reformas de la salud en Colombia. Ciencia y Tecnología. 1998;16:15-24.

27. Feo 0 . La salud pública en los procesos de la reforma y las funciones esenciales de la salud pública. Revista Facultad Nacional de Salud Pública. 2004;22:61-71. 